\title{
Using biological samples in epidemiological research on drugs of abuse
}

\author{
Hallvard Gjerde, Elisabeth Leere Øiestad and Asbjørg S. Christophersen \\ Division of Forensic Medicine and Drug Abuse Research, Norwegian Institute of Public Health, \\ P. O. Box 4404 Nydalen, 0403 Oslo \\ Correspondence: Hallvard Gjerde $\quad$ E-mail: hallvard.gjerde@fhi.no Telephone: +47 21077953
}

\begin{abstract}
Blood, oral fluid (saliva), urine and hair are the most commonly used biological matrices for drug testing in epidemiological drug research. Other biological matrices may also be used for selected purposes. Blood reflects recent drug intake and may be used to assess impairment. Oral fluid reflects drug presence in blood and thereby also recent intake, but drug concentrations in this matrix cannot be used to accurately estimate concentrations in blood. Urine reflects drug use during the last few days and in some cases for a longer period, but does not indicate the dose size or frequency of use. Hair reflects drug use during several months, but is a poor matrix for detecting use of cannabis. If using a single drug dose, this can be detected in blood and urine if the sample is taken within the detection timeframes, in most cases also in oral fluid. Single drug use is most often insufficient for producing a positive test result in a sample of hair. For cocaine and amphetamine, weekly use may be needed, while for cannabis a positive result is not guaranteed even after daily use. Refusal rates are lowest for oral fluid and highest for blood and hair samples. The analytical costs are lowest for urine and highest for hair. Combined use of questionnaires/interviews and drug testing detects more drug use than when using only one of those methods and is therefore expected to give more accurate data.
\end{abstract}

\section{INTRODUCTION}

Epidemiological studies of alcohol and drug use are most often performed by collecting self-reported data. A major difficulty with this approach is under-reporting actual use (1-14). Both the frequency and magnitude may be under-reported, and the scale of under-reporting may vary from one substance to another and may also be related to nationality, socio-economic status and other factors. For these reasons and the fact that more sensitive and accurate analytical methods have been developed, the analysis of biological specimens in epidemiological drug research has increased during the last decades, either replacing the use of questionnaires, or used as a supplement (7-14).

While under-reporting may be an issue when using questionnaires or interviews, a high refusal rate may be a problem when collecting biological samples. Therefore, the study design and the information given to participants are of great importance in order to get as many individuals to participate as possible. Refusal rates are discussed for each types of specimen in this overview.

The cost for collecting and analysing biological samples is higher than using questionnaires and may therefore be a limiting factor when deciding the number of study participants.

Ethical and legal aspects must be taken into consideration when planning a study where biological samples are to be collected. Participants must give informed consent to the use of collected samples. If the study is not anonymous, e.g. because data from registries will be used in addition to results from questionnaires and analytical tests, the legal aspects must be assessed and the necessary permissions must be obtained. If the study is not anonymous the participation rate may be lower than in an anonymous study, and the results may therefore be less representative for the studied cohort.

\section{Type of biological specimen}

A drug may be detected in any body fluid or tissue. The primary choice of biological specimen for drug analysis depends on several issues: purpose of the sampling, time interval to study, willingness to provide a voluntary sample, ease of sampling, cost of sample preparation and analysis, drug concentrations in the sample, and drug stability (15-17). When a drug is present in blood, the drug will also be present in oral fluid due to the equilibrium between body fluids; however, the concentration may in some cases be very low, sometimes lower than the analytical detection limit or cutoff concentration in oral fluid, so a positive finding is not guaranteed. A minute fraction of the drug will be deposited in growing hair. The drug will also be metabolised by the body to inactive compounds which can be analysed. Ultimately, the drug and metabolites will be eliminated by excretion in urine and faeces.

\section{Cutoff concentrations and detection times}

The detection timeframe for a drug is the average length of time after single use the drug can be detected in a biological matrix. This depends on the elimination rate for the drug combined with the analytical cutoff concentration (the drug concentration above which a finding is regarded as positive). If the drug is detected, but the concentration is below the cutoff, it will be regar- 
ded as negative (not found). If the cutoff concentration is high, a sample will remain positive for the drug for a shorter time than if the cutoff concentration is low.

The maximum drug detection timeframes for different biological matrices are indicated in Table 1 . The average detection timeframe in oral fluid is approximately as in blood if equivalent cutoff concentrations are used (18). The presence of a drug in blood is most often accompanied with detectable concentrations in oral fluid, but not always. The detection time in urine may be significantly longer, depending on the elimination rate from blood, urine elimination, and any accumulation in body fat, which is an important factor for cannabis detection. The detection timeframe in hair can be several months if the drug is efficiently incorporated in growing hair and if the drug is not degrading significantly over time.

The cutoff concentration must be larger than the detection limit for the analytical method, which is the lowest quantity of a substance that can be distinguished from the absence of that substance within a stated confidence limit. The detection limit is determined for each drug separately and is related to chemical properties of the substance, type of analytical method and analytical equipment. Often also pharmacological properties are taken into concern when determining the cutoff concentration, e.g. to avoid that minute drug concentrations reflecting use of a medicinal drug several days ago, which has no therapeutic relevance, leads to a positive finding.

Some approximate detection times for individual drugs in blood, urine and oral fluid after single use have been published (19). Cocaine and/or its principal metabolite benzoylecgonine can be detected in blood and oral fluid for up to about eight hours, in urine for a few days, and single use may in some cases be detectable in hair for several months. On the other hand, cannabis can be detected in blood and oral fluid for up to about 24 hours, in urine for a few days after single use and up to several weeks in chronic users, but can hardly be detected in hair if used only a few times each week. Thus, when choosing a biological specimen it is important to consider the type of drug in addition to the time period to be studied: recent use, which is most relevant in studies of drug use that may cause impairment at time of sample collection (e.g. among employees or drivers), or any drug use during the past week or months.

\section{False negative and positive results}

A false negative result is an analytical finding below the cutoff when the true concentration of the substance is above the cutoff concentration, and a false positive result is an analytical finding above the cutoff when the true concentration is below the cutoff or absent in the sample. For non-specific methods, like immunological assays, false positive results are commonly observed and may be obtained because of the presence of other compounds than the one being analysed. This may be a psychoactive substance, an inactive metabolite or a completely unrelated substance. Positive findings when using immunological methods should be confirmed using chromatographic methods, preferably with mass spectrometric (MS) detection.

If using an analytical method with good specificity, like a chromatographic method with MS detection, false identification of a drug is very rarely observed.

\section{Interpretation of results}

A correct interpretation of an analytical result is also important. A common situation is the detection of morphine after use of codeine because morphine is a metabolite of codeine. Another, but more rarely occurring situation is that the L-isomers of methamphetamine and amphetamine can be detected as metabolites of selegiline, which is used for the treatment of Parkinson's disease, dementia and depression. Most chromatographic methods, including those with MS detection, are not able to distinguish between those Land D-isomers; the latter ones are the illegally used substances.

\section{Specimen manipulation}

Samples of blood, oral fluid and hair are normally taken by trained personnel in close contact with the subject, so the possibility of adulteration is practically negligible. Urine is easier to adulterate because sampling is performed in a bathroom or toilet, often in some distance from the observing personnel. The sample can be manipulated either by diluting with water or an aqueous mixture, by adding chemical substances to mask the presence of drugs, by substituting the sample with clean or artificial urine, or by intentionally adding a drug.

\section{BiOLOGICAL MATERIALS}

An overview of biological matrices discussed in this paper with some advantages and disadvantages are presented in Table 1.

\section{Blood}

Blood (including serum and plasma) is the only biological specimen, except for cerebrospinal fluid, which reflects the drug concentration in the brain. Therefore, analysis of a blood sample is required if studying drug impairment. In that case, the drug concentration should be used in combination with any recorded signs of impairment and information about drug use history.

Blood samples may also be suitable for epidemiological studies in cases where samples are taken for other purposes, e.g. for patients admitted to hospital for treatment. Patients must then give informed consent to the use of blood samples for such purposes.

\section{Sampling}

Blood samples must be collected by qualified personnel. Vacuum tubes or syringes are normally used, and usually $5-10 \mathrm{ml}$ blood is collected. The blood tubes 
Table 1. Some advantages and disadvantages for different biological matrices.

\begin{tabular}{lllll}
\hline & Blood & Oral fluid & Urine & Hair \\
\hline Maximum drug detection period $^{1}$ & $1-2$ days & $1-2$ days & $2-4$ days $^{3}$ & $3-6$ months \\
Intrusive sampling & Yes & No & Yes & Yes \\
Adulteration potential & None & Low & High & Medium $^{4}$ \\
Possibility for environmental contamination & No & No & No & Yes \\
Refusal rate & High & Low & Medium & High \\
Potential for negative result after drug use & Low & Medium & Low & Medium to high \\
On-site screening possible & No & Yes & Yes & No \\
Analytical costs $^{2}$ & Medium & Medium & Medium & High \\
\hline
\end{tabular}

${ }^{1}$ Approximate detection times after intake of a single dose varying from one substance to another.

${ }^{2}$ Including confirmation testing. ${ }^{3}$ Cannabis for up to several weeks. ${ }^{4}$ Hair colouring or bleaching.

should contain preservatives like potassium fluoride. Tubes with anticoagulants are used if the intention is to collect plasma or whole blood for the analyses.

The major difficulty with using blood samples is the collection process and the willingness to provide a sample. Sampling is invasive and requires qualified personnel and suitable equipment and facilities. Studies in the USA have shown an unacceptably high refusal rate of $50-60 \%$ if collecting blood, even when $50 \$$ was offered as incentive for providing a sample $(20,21)$. In a recent study of random drivers in a number of European countries, a refusal rate of $24 \%$ was observed when asking for a blood sample in Lithuania, $55 \%$ in Belgium ( $20 €$ reward), and $25 \%$ in the Netherlands ( $10 €$ reward) (22).

An evolving technology is the analysis of dried blood spots collected on a filter paper (23-25). Only $0.1 \mathrm{ml}$ blood may be required, and a large number of drugs can be analysed simultaneously. The refusal rate might be lower when using blood spots than when collecting larger blood samples in the traditional way. At present, very few laboratories have implemented the analysis of this type of samples.

\section{Analytical methods}

Analytical methods for drugs of abuse in blood have been reviewed by Kraemer and co-workers (26). Immunoassay screening methods exist for the common classes of drugs of abuse, and work well for large screening volumes. In recent years an increasing amount of designer drugs have appeared on the market and these pose a problem for immunoassay screening as they often do not give a response on traditional immunoassays for e.g. amphetamines or cannabis. Immunoassays may also be unable to detect low-dose benzodiazepines, which are commonly misused.

Sample preparation must be performed before confirmation analysis, usually by protein precipitation (PPT), liquid-liquid extraction (LLE) or solid phase extraction (SPE). The common confirmation methods for whole blood have been gas chromatography with single or tandem mass spectrometric detection (GCMS), but liquid chromatography with single or tandem mass spectrometric detection (LC-MS) is increasingly being used for the identification and quantification of a wide range of compounds in biological samples (2729). Easier sample preparation, no derivatization required, and short analysis time are the major advantages, which allow for both screening and quantitative multi-drug methods (30). Especially in epidemiologic studies of cohorts where the prevalence of positive samples is high, specific chromatographic procedures could be a good alternative for screening, because the total costs for the analytical testing may be higher when performing a combination of immunological screening and chromatographic confirmation testing of positive samples. Our laboratory has used LC-MS for simultaneous screening and quantification in epidemiological studies with fairly low drug prevalence with good results $(8,31,32)$.

Rapid on-site screening tests have so far not been developed for blood samples. However, a recent study found that a screening device for oral fluid was found to work fairly well with blood samples (33).

\section{Interpretation of findings}

Pharmacokinetic properties for drugs in blood are well documents. Therefore, a drug concentration may be used to assess the possibility of drug impairment. For medicinal drugs, the drug concentration in blood may indicate whether a therapeutic or supra-therapeutic dose has been taken.

\section{Limitations}

The length of time a psychoactive drug can be detected in blood after single use varies from a maximum of about eight hours for cocaine to a maximum of about two days for oxazepam. Therefore, blood samples are not suitable to study drug use during a wider timeframe.

Blood samples are less suitable than urine and oral fluid in general population studies due to invasive sampling and therefore higher refusal rate.

\section{Applications}

Blood samples should be used if the aim is to determine the prevalence of drug intoxication, inebriation or supra-therapeutic drug use, e.g. in studies of the use of medicinal and illegal drugs among drivers injured or 
killed in road traffic accidents $(34,35)$, in studies of drug use among patient cohorts $(36,37)$, and in studies of fatal poisoning $(38,39)$.

\section{Urine}

Urine is the most commonly used biological matrix for testing of drugs of abuse. It is easier to collect than blood, easier to analyse, and a urine sample will be positive for a drug for a significantly longer time period than blood. After a single dose of most types of drugs, the parent drug or a metabolite may be found in urine for a few days. However, regular users of cannabis may have a positive urine sample for several weeks, even a few months, after terminating cannabis use $(40,41)$.

\section{Sampling}

Urine is normally sampled under observation by medicinal or scientific personnel to reduce the possibility of adulteration. It is usually performed in a bathroom or toilet, in some cases equipped with a surveillance window so that sample collection personnel may observe the sampling process.

The refusal rate may be lower than for blood sampling. In an American study where an incentive of 10 or $20 \$$ was given for each biological sample, the refusal rate for urine was about 24\% (9).

\section{Analytical methods}

The common analytical methods for urine have been immunoassay screening combined with GC-MS confirmation (42). Immunoassays are sensitive, automated and do not require pre-treatment. The limited number of drugs that can be tested by immunoassays is a disadvantage. Several recent analytical strategies apply LC-MS as an alternative for urine screening (43-45) to increase the drug repertoire. This technique has in addition increasingly been used as a supplement to GC-MS for confirmation of drug findings $(27,28,46)$. Hydrolysis of excreted drug conjugates (e.g. glucuronides) and sample preparation by LLE or SPE is common before GC-MS analysis. For LC-MS analysis, dilution and direct injection of urine samples is possible, and in addition direct measurement of glucuronides can be performed (47).

Many on-site (e.g. dip-stick) immunological tests for drugs of abuse exist for urine. Due to the inherent possibility of false positive results, confirmation analysis should be performed also for epidemiological studies.

\section{Interpretation of findings}

A positive finding of a drug or metabolite confirms drug use during the last few days; and for cannabis possibly for a longer time period. The result does not indicate the dose used or approximate time of intake, and cannot be used to assess impairment.

\section{Limitations}

A bathroom or toilet must be available for sample collection. In some cases, the donor is unable to provide a urine sample when requested, and may have to wait for a long time before this can be done. This may cause delay of the inclusion of subjects in an epidemiological study. Urine has a high adulteration potential.

\section{Applications}

Urine samples may be analysed if the aim is to detect drug use during the latest days before sample collection, e.g. in studies of drug use among adolescents or students (48-51), drivers $(52,53)$ and worker cohorts $(11,54)$. Please note that urine samples may be positive for cannabis for a significantly longer time.

\section{Oral fluid}

Oral fluid (mixed saliva) is an easily available medium that can be collected without the intrusion of privacy. Drug findings in oral fluid reflects recent use, and is therefore a better choice than urine or hair if studying drug use that may have a pharmacological effect at time of sampling. It is difficult to adulterate.

The use of oral fluid in testing for drugs of abuse has increased during the last decades (55-57). Samples of oral fluid routinely used for workplace drug testing and for the follow-up of drug addicts undergoing treatment.

In the 1990s, many researchers believed that drug concentrations in blood or plasma could be calculated based on concentrations in oral fluid as soon as the conversion factors (oral fluid to blood concentration ratios; OF/B) had been determined experimentally. Later research has shown that this was impossible, also when collecting oral fluid in a controlled manner. Significant inter- and intra-subject variability in $\mathrm{OF} / \mathrm{B}$ ratios was observed, and the wide variation of ratios do not allow reliable estimation of drug concentrations in blood based on concentrations in oral fluid $(58,59)$.

\section{Sampling}

Several sampling devices for oral fluid are commercially available (60). The devices are composed by a sampling pad and a test tube containing a buffer with a preservative to stabilise the samples and to avoid microbiological degradation. The sampling pad may contain chemicals that stimulate salivation. The pad is placed under the tongue or between tongue and cheek for typically 2-5 minutes. Some sampling pads have an indicator which changes colour when sufficient oral fluid has been collected. Typically, $0.3-1 \mathrm{ml}$ oral fluid is collected. The use of some drugs may cause reduced flow of saliva, and therefore small sample volume. After collection is completed, the pad is transferred to the test tube.

The refusal rate for providing a sample of oral fluid can be very low. In recent studies in Norway, the refusal rate was 3-6\% $(8,22,61)$. In an American study, the refusal rate was $8-11 \%$ when giving an incentive of 10 or $20 \$(9)$.

\section{Analytical methods}

On-site screening devices using immunological testing are available for the major drugs (57). Immunoassays for oral fluid are commonly used in e.g. commercial work-place testing (62). As for immunological methods 
for blood, the types of drugs that can be detected by immunological methods are limited. In addition, difficulties with detection of cannabis have frequently been reported with immunological methods for oral fluid (63). More sensitive chromatographic methods for screening have been developed to solve those problems.

Sample preparation is commonly performed by LLE or SPE. Dilution or PPT might be performed for expectorated oral fluid, while more extensive sample preparation is necessary for oral fluid collected with commercial sampling kits which contains preservatives, surfactants and in some cases dyes which might interfere with instrumental analysis. Confirmatory analysis of oral fluid is most often performed with chromatographic - MS techniques (57) due to very good specificity and sensitivity. GC-MS methods have been published for all common drugs of abuse in oral fluid. As oral fluid sample volume is limited, multicomponent chromatographic assays are emerging as a solution for both screening and confirmation. While multi-component GC-methods have been published, the requirement of different derivatization agents for different drug classes gives quite complex sample preparation (64). Most new methods are therefore based on LC-MS (57).

\section{Interpretation of findings}

A positive drug finding in oral fluid reflects drug presence in blood and confirms drug use typically within the last 1-2 days, varying from one drug to another (19). In chronic users who terminate their drug use, an oral fluid sample may be positive for the drug for a significantly longer time than after single intake; this varies from one drug to another. The analytical result does not indicate impairment, the dose used or approximate time of intake. A negative drug finding in oral fluid does not absolutely exclude the possibility of recent drug intake. Drug concentrations in oral fluid cannot be used to accurately estimate drug concentrations in blood $(58,59)$. When studying a cohort, the prevalence of drug concentrations in blood above selected concentrations limits may be estimated based on the analysis of oral fluid $(65,66)$.

\section{Limitations}

The primary limitations are that drug findings reflect only recent drug use. Even though a positive drug finding in oral fluid reflects drug presence in blood, the drug concentration in oral fluid cannot be used to accurately estimate the drug concentration in blood.

\section{Applications}

Oral fluid is suitable if the aim is to study the prevalence of drug use during the last 1-2 days before sample collection, e.g. in studies of drug use in the general population (9), among random drivers $(22,31,67-69)$ or workers $(8,70)$.

\section{Hair}

Hair testing has a wider detection timeframe than urine, blood and oral fluid. Hair samples show good stability during storage at room temperature, and they are easy to ship. By sectional analysis of hair samples, a historical overview of drug intake during the last several months, perhaps up to a year or more, may be obtained (71). One centimetre of hair corresponds to about one month of growth, and drug findings in one centimetre thus represented drug intake during a month. However, hair testing cannot always detect drug use within the last week, because drug containing hair appears above the skin surface a few days after it was incorporated within the hair follicle (71).

The exact mechanism for drug incorporation into hair is not known, but it is proposed that drugs can enter from blood during hair formation, from sebum and sweat, and from the external environment (72).

\section{Sampling}

Sampling is less intrusive than blood sampling. Hair is best collected from the back of the head with approximately a pencil width and a weight of about $200 \mathrm{mg}$. The sample should be wrapped in aluminium foil and stored under dry conditions at room temperature. Hair has a potential for environmental contamination. Therefore, hair samples must be properly decontaminated by washing with different solvents before drug analysis is performed.

The refusal rate when collecting hair may be fairly large, mainly for cosmetic reasons. In an American study of drug use in a high-risk community sample of adults from Chicago, hair samples were obtained from only $56 \%$ of the participants (10). In a later study, the refusal rate was $14-16 \%$ when giving an incentive of 10 or 20 US\$, however, $21 \%$ had insufficient hair for sampling (9). Thus, suitable hair samples were obtained from only about $64 \%$ in the latter study.

\section{Analytical methods}

A hair segment - an appropriate length of hair - must be cut from the hair sample. Shorter lengths represent shorter time intervals. Washing with solvents to remove possible external contamination is usually performed. This can however affect the extraction efficiencies of incorporated drugs and must be considered when interpreting quantitative results. Pretreatment steps must be applied to extract drugs from hair as they are firmly enclosed in the hair structure and partly bound to proteins, melanin or lipids of the cell membrane complex (73). The hair is often cut into small pieces or pulverized by grinding. Methanolic extraction $(5-18 \mathrm{~h})$ in an ultrasonic bath can be used for most drugs. Other possibilities, depending on type of drug, are acidic extraction, alkaline digestion, enzymatic hydrolysis or incubation with neutral or slightly acidic aqueous buffer. In aqueous hair extracts hydrolysis of cocaine to benzoylecgonine and heroine/6-monoacetylmorphine to morphine can occur. Heroin itself has therefore not been found in aqueous hair extracts (73). Further clean-up by LLE or SPE might be necessary before analysis.

Analytical methods for drugs of abuse in hair have been reviewed by Wada and co-workers (74). Immunoassay methods for hair extracts exist (75), but as hair 
analysis requires sensitive and selective methods GCMS and LC-MS are common analysis methods. Some applications does in addition use LC coupled to fluorescence or chemiluminescence (74). Sensitive multianalyte methods for drugs of abuse have been published $(76,77)$, making hair screening somewhat less labour intensive.

\section{Interpretation of findings}

Interpretation of analytical results has been mentioned as "the most serious pitfalls of hair analysis" (75). There are large individual variations in drug incorporation and drug concentrations in hair. In addition, cosmetic treatment may reduce drug concentrations significantly. If a drug is found in a hair sample that is properly treated to eliminate environmental contamination and an analytical method based on mass spectrometry is used, drug intake is confirmed. However, if a drug is not found, drug intake cannot be excluded. Intake of a single dose of cocaine, codeine, benzodiazepines and GHB has been detected in hair (78-80). In a study of 100 users of amphetamines, only $56 \%$ had positive hair sample (81), indicating that a negative finding does not exclude the possibility of drug use. For cannabis a positive result is not guaranteed even after daily use (82). In general, hair samples are very well suited for the detection of cocaine use, fairly well suited for detection of amphetamines and benzodiazepines, and not very well suited for detection of cannabis use.

If hair segments are analysed, variations in hair growth between individuals may lead to inaccurate interpretation of the time for drug intake.

\section{Limitations}

Hair treatments, like colouring or bleaching, can reduce drug concentrations significantly (83). Frequent washing can also reduce drug concentrations somewhat. It has also been claimed that drug concentrations might be related to hair colour (83), thus also introducing an ethnical bias. Hair is not a well suited specimen for detecting use of cannabis. The drug concentration does not accurately reflect the taken dose (84), however, a semi-quantitative relationship has been reported for codeine and cocaine in a controlled study (85).

An important limitation of hair testing is the high analytical cost. It is therefore not very well suited for mass screening or studies of large cohorts.

\section{Applications}

Hair samples are suitable for the detection of drug use during the last several months, e.g. in studies of drug use in the general population (4), among students (86, 87 ), patients $(6,13)$, or drug addicts $(1,88)$. The analysis is time-consuming and expensive; therefore hair samples are best suited for studying cohorts with high proportion of drug users.

\section{Other biological materials}

Drugs may be detected in sweat and reflects recent use. Sweat pads or wipes are simple adsorptive pads that are moistened with ethanol or isopropanol and used to wipe over the forehead or any other skin to collect moisture and sebum from the skin (89). Sweat patches, similar to small bandages, consist of an adhesive layer on a thin transparent film of surgical dressing to which an adsorbent cotton or cellulose pad is attached (90). The patch is worn on the chest, back or biceps for a period and then removed and analysed. Fast patches $(91,92)$ are designed to be applied for up to 30 minutes and rely on heating to induce sweating for specimen collection. Other types are designed for collecting sweat for up to 14 days $(89,93,94)$, thus, the cumulative drug exposure may be studied. The latter type is not suitable for general population studies, but may be used to study relapse among drug addicts during treatment.

Breath is mostly used for the analysis of alcohol. However, recent sensitive technologies allow the detection of amphetamines, methadone, and other substances. Special equipment is needed, and at present this is probably only at its initial stage of development (95-97).

Nails may be used instead of hair to detect drug use $(98,99)$.

To analyse drug exposure of newborn babies for drugs used by their mothers, meconium (the first faecal matter passed by a neonate), umbilical cord tissue or blood, vernix caseosa (a thick, white lipid and cell mixture that covers the fetus) and amniotic fluid may be analysed to study drug use during the last part of the pregnancy $(100,101)$. After the child is born, breast milk may be analysed to detect drug use (100).

\section{Combined use of self-reporting with biological testing}

Usually, neither the use of questionnaires or interviews nor the analysis of biological samples can provide accurate information on past drug use in a cohort. The only exception is the testing of blood or urine samples to detect very recent drug use. A number of reports have concluded that the combination of self-reported drug use with testing of biological samples can show higher and more accurate prevalence of drug use (7,11-14). In spite of the fact that the validity of self-reported drug use may not be very good, self-report is needed when studying drug use history, including frequency and quantity of use, which are data that biological testing cannot provide.

Fendrich and coworkers (5) found in a general population survey conducted in Chicago that many people who were reluctant to disclose drug use in a survey had little problem with allowing researchers to collect biological samples that confirmed drug use. In total, analysis of oral fluid, urine and hair revealed that $16.6 \%, 11.6 \%$ and $2.1 \%$ had used cannabis, cocaine and heroin, respectively. Self-reports indicated that about $23.3 \%, 4.2 \%$ and $0.3 \%$, respectively, had used those drugs during the past three months. Thus, the under-reporting was particularly large for cocaine and heroin. Similar results were found in studies of worker 
Table 2: Assessment of the accuracy potential for testing of biological samples alone and in combination with questionnaires. Number of stars indicates the accuracy potential, no star indicates unsuitability.

\begin{tabular}{lccccc}
\hline & Last $6 \mathrm{~h}$ & Last 24 h & Last week & Last month & Last several months \\
\hline Blood & $* * * * * *$ & $* * * *$ & - & - & - \\
Oral fluid & $* * * * *$ & $* * * *$ & - & - & - \\
Urine & $* * * *$ & $* * * * * *$ & $* * *$ & - & - \\
Hair & - & - & - & $* * *$ & $* * *$ \\
Blood+questionnaire & $* * * * *$ & $* * * * *$ & - & - & - \\
Oral fluid+questionnaire & $* * * * *$ & $* * * * *$ & - & - & - \\
Urine+questionnaire & $* * * *$ & $* * * * * *$ & $* * * *$ & - & - \\
Hair+questionnaire & - & - & - & $* * * *$ & $* * * *$ \\
Urine+hair+questionnaire & - & - & - & $* * * *$ & $* * * *$ \\
\hline
\end{tabular}

cohorts $(11,12)$, for psychiatric patients $(6,13)$, crack cocaine users (102) and adolescents $(7,14)$.

One limitation of the combined use of questionnaires and self-reported use is the detection window for the different biological matrices. If asking about drug use during the last month, neither samples of blood, oral fluid or urine reflects the same time period, but will provide additional data. Samples of hair may be cut to cover the same period, but will mostly reflect frequent use, not low to moderate drug use, varying from one substance to another. However, in total, the analysis of biological samples will provide additional information about drug use, and the final results will be more accurate than when using only self-reported data.

An assessment of the potentials for combining selfreports with analysis of different types of biological samples is presented in Table 2.

\section{Conclusion}

The type of specimen and the cutoff concentration must be taken into consideration when planning an epidemiological study on drug use. If recent intake is being studied (during the last 6-48 hours), blood or oral fluid should be collected. If drug use during the last few days is being studied, urine samples are better. If use during the last few months is being studied, hair samples are the best, but unreliable for cannabis. The most reliable specimen types are blood and urine; drug intake will give positive results for those two specimen types within the detection time windows, but not always for oral fluid and hair.

The refusal rates and cost of analysis should also be taken into consideration. Refusal rates are lowest for the collection of oral fluid and highest for blood and hair samples. The costs are lowest for urine and highest for hair.

Analysis of biological samples will not be able to detect all drug use, except for blood samples taken shortly after drug intake, or urine samples taken within a certain timeframe after use. For oral fluid and hair, a negative test result does not prove that a drug was not taken. Therefore, the best strategy when doing epidemiological studies of drug use may be to combine the use of biological samples with questionnaires or interviews. The combined prevalence of drug use will be closer to the true figure, and higher than if just one of the techniques is used alone.

\section{REFERENCES}

1. Magura S, Kang SY. Validity of self-reported drug use in high risk populations: a meta-analytical review. Subst Use Misuse 1996; 31 (9): 1131-1153.

2. Harrison L, Hughes A. The validity of self-reported drug use: improving the accuracy of survey estimates. NIDA Research Monograph no. 167. Rockville MD: National Institute on Drug Abuse, 1997.

3. Mieczkowski T, Newel R, Wraight B. Using hair analysis, urinalysis, and self-reports to estimate drug use in a sample of detained juveniles. Subst Use Misuse 1998; 33 (7): 1547-1567.

4. Colon HM, Robles RR, Sahai H. The validity of drug use responses in a household survey in Puerto Rico: comparison of survey responses of cocaine and heroin use with hair tests. Int J Epidemiol 2001; 30 (5): 1042-1049.

5. Fendrich M, Johnson TP, Wislar JS, Hubbell A, Spiehler V. The utility of drug testing in epidemiological research: results from a general population survey. Addiction 2004; 99 (2): 197-208.

6. Musshoff F, Driever F, Lachenmeier K, Lachenmeier DW, Banger M, Madea B. Results of hair analyses for drugs of abuse and comparison with self-reports and urine tests. Forensic Sci Int 2006; 156 (2-3): 118-123.

7. Williams RJ, Nowatzki N. Validity of adolescent self-report of substance use. Subst Use Misuse 2005; 40 (3): 299 311.

8. Gjerde H, Christophersen AS, Moan IS et al. Use of alcohol and drugs by Norwegian employees: a pilot study using questionnaires and analysis of oral fluid. J Occup Med Toxicol 2010; 5: 13.

9. Fendrich M, Johnson TP, Wislar JS, Hubbell A. Drug test feasibility in a general population household survey. Drug Alcohol Depend 2004; 73 (3): 237-250. 
10. Fendrich M, Johnson TP, Sudman S, Wislar JS, Spiehler V. Validity of drug use reporting in a high-risk community sample: a comparison of cocaine and heroin survey reports with hair tests. Am J Epidemiol 1999; 149 (10): 955962.

11. Bharucha-Reid R, McCann D, Schork MA, Foxman B. A comparison of self-reported drug use with a urine drug screen in a working population. Environ Clin Psychopharmacol 1995; 3 (3): 280-286.

12. Hersch RK, McPherson TL, Cook RF. Substance use in the construction industry: a comparison of assessment methods. Subst Use Misuse 2002; 37 (11): 1331-1358.

13. Swartz MS, Swanson JW, Hannon MJ. Detection of illicit substance use among persons with schizophrenia by radioimmunoassay of hair. Psychiatr Serv 2003; 54 (6): 891-895.

14. Delaney-Black V, Chiodo LM, Hannigan JH et al. Just say "I don't": lack of concordance between teen report and biological measures of drug use. Pediatrics 2010; 126 (5): 887-893.

15. Dolan K, Rouen D, Kimber J. An overview of the use of urine, hair, sweat and saliva to detect drug use. Drug Alcohol Rev 2004; 23 (2): 213-217.

16. Paterson S. Choice of specimen for analysis for verifying a peron's drug status. J Subst Use 2008; 13 (4): $239-246$.

17. Vearrier D, Curtis JA, Greenberg MI. Biological testing for drugs of abuse. EXS 2010; 100: 489-517.

18. Samyn N, Verstraete A, van Haeren C, Kintz P. Analysis of drugs of abuse in saliva. Forensic Sci Rev $1999 ; 11$ (1): 2-17.

19. Verstraete AG. Detection times of drugs of abuse in blood, urine, and oral fluid. Ther Drug Monit $2004 ; 26$ (2): 200-205.

20. Lacey JH, Kelley-Baker T, Furr-Holden D, Brainard K, Moore C. Pilot test of new roadside survey methodology for impaired driving. DOT HS 810 704. Washington: National Highway Traffic Safety Administration, 2007.

21. Lacey JH, Kelley-Baker T, Furr-Holden D et al. 2007 national roadside survey of alcohol and drug use by drivers drug results. DOT HS 811 249. Washington: National Highway Safety Administration, 2009.

22. Houwing S, Hagenzieker M, Mathijssen R et al. Prevalence of alcohol and other psychoactive substances in drivers in general traffic. Part 1: General results. Leidschendam: SWOV, 2011.

23. Henderson LO, Powell MK, Hannon WH et al. An evaluation of the use of dried blood spots from newborn screening for monitoring the prevalence of cocaine use among childbearing women. Biochem Mol Med 1997; 61 (2): 143-151.

24. Garcia BR, Henseler J, Mattern R, Skopp G. Determination of morphine and 6-acetylmorphine in blood with use of dried blood spots. Ther Drug Monit 2008; 30 (6): 733-739.

25. Langel K. Dried blood spot as a sample matrix for drug analysis - a validated method for screening and quantitation of 23 drugs of abuse by gas chromatography-mass spectrometry. Abstracts of the 19th International Conference on Alcohol, Drugs and Traffic Safety (T2010) in Oslo, Norway, August 22-26, 2010, Oslo: Norwegian Institute of Public Health, 2010: 202.

26. Kraemer T, Paul L. Bioanalytical procedures for determination of drugs of abuse in blood. Anal Bioanal Chem 2007; 388 (7): 1415-1435.

27. Peters FT. Recent advances of liquid chromatography-(tandem) mass spectrometry in clinical and forensic toxicology. Clin Biochem 2011; 44 (1): 54-65.

28. Maurer H. Current role of liquid chromatography-mass spectrometry in clinical and forensic toxicology. Anal Bioanal Chem 2007; 388 (7): 1315-1325.

29. Saint-Marcoux F, Sauvage FL, Marquet P. Current role of LC-MS in therapeutic drug monitoring. Anal Bioanal Chem 2007; 388 (7): 1327-1349.

30. Maurer HH. Multi-analyte procedures for screening for and quantification of drugs in blood, plasma, or serum by liquid chromatography-single stage or tandem mass spectrometry (LC-MS or LC-MS/MS) relevant to clinical and forensic toxicology. Clin Biochem 2005; 38 (4): 310-318.

31. Gjerde H, Normann PT, Pettersen BS et al. Prevalence of alcohol and drugs among Norwegian motor vehicle drivers: a roadside survey. Accid Anal Prev 2008; 40 (5): 1765-1772.

32. Gjerde H, Christophersen AS, Normann PT et al. Country report Norway. In: Houwing S et al, ed. Prevalence of alcohol and other psychoactive substances in drivers in general traffic. Part II: Country reports. DRUID deliverable D2.2.3, Leidschendam: SWOV Institute for Road Safety Research, 2011: 152-163.

33. Arroyo A, Sanchez M, Palahi M, Barbal M, Marron MA, Mora A. Applicability of an on-site test for its use in postmortem blood. Leg Med (Tokyo) 2011; 13 (5): 240-244.

34. Gonzalez-Wilhelm L. Prevalence of alcohol and illicit drugs in blood specimens from drivers involved in traffic law offenses. Systematic review of cross-sectional studies. Traffic Inj Prev 2007; 8 (2): 189-198.

35. Isalberti C, Van der Linden T, Legrand SA et al. Prevalence of alcohol and other psychoactive substances in injured and killed drivers. DRUID Deliverable 2.2.5. Ghent: University of Ghent, 2011.

36. Vitale S, van de Mheen D. Illicit drug use and injuries: A review of emergency room studies. Drug Alcohol Depend 2006; 82 (1): 1-9.

37. Cherpitel CJ. Alcohol and injuries: a review of international emergency room studies since 1995. Drug Alcohol Rev 2007; 26 (2): 201-214.

38. Hall AJ, Logan JE, Toblin RL et al. Patterns of abuse among unintentional pharmaceutical overdose fatalities. JAMA 2008; 300 (22): 2613-2620.

39. Simonsen KW, Normann PT, Ceder G et al. Fatal poisoning in drug addicts in the Nordic countries in 2007. Forensic Sci Int 2011; 207 (1-3): 170-176.

40. Johansson E, Halldin MM. Urinary excretion half-life of delta 1-tetrahydrocannabinol-7-oic acid in heavy marijuana users after smoking. J Anal Toxicol 1989; 13 (4): 218-223. 
41. Ellis GM, Mann MA, Judson BA, Schramm NT, Tashchian A. Excretion patterns of cannabinoid metabolites after last use in a group of chronic users. Clin Pharmacol Ther 1985; 38 (5): 572-8.

42. Chaturvedi AK, Liu RH. Substance misuse - Urine analysis. In: Payne-James J, ed. Encyclopedia of Forensic and Legal Medicine, Oxford: Elsevier, 2005: 171-183.

43. Wissenbach D, Meyer M, Remane D, Philipp A, Weber A, Maurer H. Drugs of abuse screening in urine as part of a metabolite-based LC-MSn screening concept. Anal Bioanal Chem 2011; 400 (10): 3481-9.

44. Nordgren HK, Beck O. Multicomponent screening for drugs of abuse - Direct analysis of urine by LC-MS-MS. Ther Drug Monit 2004; 26 (1): 90-97.

45. Eichhorst JC, Etter ML, Rousseaux N, Lehotay DC. Drugs of abuse testing by tandem mass spectrometry: a rapid, simple method to replace immunoassays. Clin Biochem 2009; 42 (15): 1531-1542.

46. Pizzolato TM, de Alda MJL, Barcelo D. LC-based analysis of drugs of abuse and their metabolites in urine. TracTrends in Analytical Chemistry 2007; 26 (6): 609-624.

47. Hegstad S, Khiabani HZ, Oiestad EL, Berg T, Christophersen AS. Rapid quantification of buprenorphine-glucuronide and norbuprenorphine-glucuronide in human urine by LC-MS-MS. J Anal Toxicol 2007; 31 (4): 214-219.

48. Quiroga PN, Panzuto RI, Alvarez GB et al. First analytical chemistry study on drug abuse in the Buenos Aires (Argentina) University students. Farmaco 1998; 53 (6): 389-394.

49. Thevis M, Sauer M, Geyer H, Sigmund G, Mareck U, Schanzer W. Determination of the prevalence of anabolic steroids, stimulants, and selected drugs subject to doping controls among elite sport students using analytical chemistry. J Sports Sci 2008; 26 (10): 1059-1065.

50. Garcia-Jimenez S, Heredia-Lezama K, Bilbao-Marcos F, Fuentes-Lara G, Monroy-Noyola A, Deciga-Campos M. Screening for marijuana and cocaine abuse by immunoanalysis and gas chromatography. Ann N Y Acad Sci 2008; 1139: 422-425.

51. Kapusta ND, Ramskogler K, Hertling I et al. Epidemiology of substance use in a representative sample of 18-yearold males. Alcohol Alcohol 2006; 41 (2): 188-192.

52. Maes V, Samyn N, Willekens M, De Boeck G, Verstraete AG. Stupéfiants et conduite automobile - les actions réalisées en Belgique [Drugs and driving - the Belgian experience]. Ann Toxicol Anal 2003; 15 (2): 128-137.

53. Sugrue M, Seger M, Dredge G et al. Evaluation of the prevalence of drug and alcohol abuse in motor vehicle trauma in south western Sydney. Aust N Z J Surg 1995; 65 (12): 853-856.

54. Labat L, Fontaine B, Delzenne $\mathrm{C}$ et al. Prevalence of psychoactive substances in truck drivers in the Nord-Pas-deCalais region (France). Forensic Sci Int 2008; 174 (2-3): 90-94.

55. Choo RE, Huestis MA. Oral fluid as a diagnostic tool. Clin Chem Lab Med 2004; 42 (11): 1273-1287.

56. Drummer OH. Drug testing in oral fluid. Clin Biochem Rev 2006; 27 (3): 147-159.

57. Bosker WM, Huestis MA. Oral fluid testing for drugs of abuse. Clin Chem 2009; 55 (11): 1910-1931.

58. Wille SMR, Raes E, Lillsunde P et al. Relationship between oral fluid and blood concentrations of drugs of abuse in drivers suspected of DUID. Ther Drug Monit 2009; 31 (4): 511-519.

59. Gjerde H, Mordal J, Christophersen AS, Bramness JG, Mørland J. Comparison of drug concentrations in blood and oral fluid collected with the Intercept sampling device. J Anal Toxicol 2010; 34 (4): 204-209.

60. Langel K, Engblom C, Pehrsson A, Gunnar T, Ariniemi K, Lillsunde P. Drug testing in oral fluid-evaluation of sample collection devices. J Anal Toxicol 2008; 32 (6): 393-401.

61. Gjerde H, Christophersen AS, Normann PT et al. Analysis of alcohol and drugs in oral fluid from truck drivers in Norway. Traffic Inj Prev 2011 (in press).

62. Townsend S, Fanning L, O'Kennedy R. Salivary analysis of drugs - potential and difficulties. Anal Lett $2008 ; 41$ (6): 925-948.

63. Blencowe T, Pehrsson A, Lillsunde P et al. An analytical evaluation of eight on-site oral fluid drug screening devices using laboratory confirmation results from oral fluid. Forensic Sci Int 2011; 208 (1-3): 173-179.

64. Langel K, Gunnar T, Ariniemi K, Rajamaki O, Lillsunde P. A validated method for the detection and quantitation of 50 drugs of abuse and medicinal drugs in oral fluid by gas chromatography-mass spectrometry. $J$ Chromatogr $B$ Biomed Sci Appl 2011; 879 (13-14): 859-870.

65. Gjerde H, Verstraete A. Can the prevalence of high blood drug concentrations in a population be estimated by analysing oral fluid? A study of tetrahydrocannabinol and amphetamine. Forensic Sci Int 2010; 195 (1-3): 153-159.

66. Gjerde H, Verstraete AG. Estimating equivalent cutoff thresholds for drugs in blood and oral fluid using prevalence regression: a study of tetrahydrocannabinol and amphetamine. Forensic Sci Int 2011; 212 (1-3): e26-e30.

67. Behrensdorff I, Steentoft A. Medicinal and illegal drugs among Danish car drivers. Accid Anal Prev $2003 ; 35$ (6): 851-860.

68. Wylie FM, Torrance H, Seymour A, Buttress S, Oliver JS. Drugs in oral fluid Part II. Investigation of drugs in drivers. Forensic Sci Int 2005; 150 (2-3): 199-204.

69. Drummer OH, Gerostamoulos D, Chu M, Swann P, Boorman M, Cairns I. Drugs in oral fluid in randomly selected drivers. Forensic Sci Int 2007; 170 (2-3): 105-110.

70. Cook RF, Bernstein AD, Andrews CM. Assessing drug use in the workplace: a comparison of self-report, urinalysis, and hair analysis. NIDA Res Monogr 1997; 167: 247-272.

71. Pragst F, Rothe M, Spiegel K, Sporkert F. Illegal and therapeutic drug concentrations in hair segments - a timetable of drug exposure? Forensic Sci Rev 1998; 10 (2): 81-111.

72. Kintz P, Villain M, Cirimele V. Hair analysis for drug detection. Ther Drug Monit 2006; 28 (3): $442-446$.

73. Musshoff F, Madea B. Analytical pitfalls in hair testing. Anal Bioanal Chem 2007; 388 (7): 1475-1494. 
74. Wada M, Ikeda R, Kuroda N, Nakashima K. Analytical methods for abused drugs in hair and their applications. Anal Bioanal Chem 2010; 397 (3): 1039-1067.

75. Pragst F, Balikova MA. State of the art in hair analysis for detection of drug and alcohol abuse. Clin Chim Acta 2006; 370 (1-2): 17-49.

76. Hegstad S, Khiabani HZ, Kristoffersen L, Kunoe N, Lobmaier PP, Christophersen AS. Drug screening of hair by liquid chromatography-tandem mass spectrometry. J Anal Toxicol 2008; 32 (5): 364-372.

77. Kronstrand R, Nystrom I, Strandberg J, Druid H. Screening for drugs of abuse in hair with ion spray LC-MS-MS. Forensic Sci Int 2004; 145 (2-3): 183-190.

78. Henderson GL, Harkey MR, Zhou C, Jones RT, Jacob P, III. Incorporation of isotopically labeled cocaine and metabolites into human hair: 1. dose-response relationships. J Anal Toxicol 1996; 20 (1): 1-12.

79. Kintz P. Bioanalytical procedures for detection of chemical agents in hair in the case of drug-facilitated crimes. Anal Bioanal Chem 2007; 388 (7): 1467-1474.

80. Kronstrand R, Forstberg-Peterson S, Kagedal B, Ahlner J, Larson G. Codeine concentration in hair after oral administration is dependent on melanin content. Clin Chem 1999; 45 (9): 1485-1494.

81. Moore C, Feldman M, Giorgi N et al. Analysis of amphetamines in hair, oral fluid and urine. Ann Toxicol Anal 2005; 17 (4): 229-236.

82. Huestis MA, Gustafson RA, Moolchan ET et al. Cannabinoid concentrations in hair from documented cannabis users. Forensic Sci Int 2007; 169 (2-3): 129-136.

83. Stout PR. Hair testing for drugs - challenges for interpretation. Forensic Sci Rev 2007; 19 (2): 69-84.

84. Harkey MR. Technical issues concerning hair analysis for drugs of abuse. NIDA Res Monogr 1995; 154: $218-234$.

85. Scheidweiler KB, Cone EJ, Moolchan ET, Huestis MA. Dose-related distribution of codeine, cocaine, and metabolites into human hair following controlled oral codeine and subcutaneous cocaine administration. J Pharmacol Exp Ther 2005; 313 (2): 909-915.

86. Kidwell DA, Blanco MA, Smith FP. Cocaine detection in a university population by hair analysis and skin swab testing. Forensic Sci Int 1997; 84 (1-3): 75-86.

87. Quintela O, Bermejo AM, Tabernero MJ, Strano-Rossi S, Chiarotti M, Lucas AC. Evaluation of cocaine, amphetamines and cannabis use in university students through hair analysis: preliminary results. Forensic Sci Int 2000; 107 (1-3): 273-279.

88. Welp EA, Bosman I, Langendam MW, Totte M, Maes RA, van Ameijden EJ. Amount of self-reported illicit drug use compared to quantitative hair test results in community-recruited young drug users in Amsterdam. Addiction 2003; 98 (7): 987-994.

89. Kidwell DA, Kidwell JD, Shinohara F et al. Comparison of daily urine, sweat, and skin swabs among cocaine users. Forensic Sci Int 2003; 133 (1-2): 63-78.

90. Kidwell DA, Holland JC, Athanaselis S. Testing for drugs of abuse in saliva and sweat. J Chromatogr B Biomed Sci Appl 1998; 713 (1): 111-135.

91. Huestis MA, Oyler JM, Cone EJ, Wstadik AT, Schoendorfer D, Joseph RE, Jr. Sweat testing for cocaine, codeine and metabolites by gas chromatography-mass spectrometry. J Chromatogr B Biomed Sci Appl 1999; 733 (1-2): 247 264.

92. Liberty HJ, Johnson BD, Fortner N, Randolph D. Detecting crack and other cocaine use with fastpatches. Addict Biol 2003; 8 (2): 191-200.

93. Liberty HJ, Johnson BD, Fortner N. Detecting cocaine use through sweat testing: multilevel modeling of sweat patch length-of-wear data. J Anal Toxicol 2004; 28 (8): 667-673.

94. Brunet BR, Barnes AJ, Choo RE, Mura P, Jones HE, Huestis MA. Monitoring pregnant women's illicit opiate and cocaine use with sweat testing. Ther Drug Monit 2010; 32 (1): 40-49.

95. Manolis A, McBurney LJ, Bobbie BA. The detection of delta 9-tetrahydrocannabinol in the breath of human subjects. Clin Biochem 1983; 16 (4): 229-233.

96. Beck O, Leine K, Palmskog G, Franck J. Amphetamines detected in exhaled breath from drug addicts: A new possible method for drugs-of-abuse testing. J Anal Toxicol 2010; 34 (5): 233-237.

97. Beck O, Sandqvist S, Eriksen P, Franck J, Palmskog G. Determination of methadone in exhaled breath condensate by liquid chromatography-tandem mass spectrometry. J Anal Toxicol 2011; 35 (3): 129-133.

98. Palmeri A, Pichini S, Pacifici R, Zuccaro P, Lopez A. Drugs in nails: physiology, pharmacokinetics and forensic toxicology. Clin Pharmacokinet 2000; 38 (2): 95-110.

99. Irving RC, Dickson SJ. The detection of sedatives in hair and nail samples using tandem LC-MS-MS. Forensic Sci Int 2007; 166 (1): 58-67.

100. Gallardo E, Queiroz JA. The role of alternative specimens in toxicological analysis. Biomed Chromatogr $2008 ; 22$ (8): 795-821.

101. Gray T, Huestis M. Bioanalytical procedures for monitoring in utero drug exposure. Anal Bioanal Chem 2007; 388 (7): $1455-1465$.

102. Schumacher JE, Milby JB, Raczynski JM et al. Validity of self-reported crack cocaine use among homeless persons in treatment. J Subst Abuse Treat 1995; 12 (5): 335-339. 